\title{
Comparison of self-administration behavior and responsiveness to drug-paired cues in rats running an alley for intravenous heroin and cocaine
}

\author{
Zu-In Su • Jennifer Wenzel • Rebeccah Baird • \\ Aaron Ettenberg
}

Received: 12 August 2010 / Accepted: 28 October 2010 /Published online: 18 November 2010

(C) The Author(s) 2010. This article is published with open access at Springerlink.com

\begin{abstract}
Rationale Evidence suggests that responsiveness to a drugpaired cue is predicted by the reinforcing magnitude of the drug during prior self-administration. It remains unclear, however, if this principle holds true when comparisons are made across drug reinforcers.

Objective The current study was therefore devised to test the hypothesis that differences in the animals' responsiveness to a cocaine- or heroin-paired cue presented during extinction would reflect differences in the patterns of prior cocaine and heroin runway self-administration.

Methods Rats ran a straight alley for single intravenous injections of either heroin $(0.1 \mathrm{mg} / \mathrm{kg} / \mathrm{inj})$ or cocaine $(1.0 \mathrm{mg} / \mathrm{kg} / \mathrm{inj})$ each paired with a distinct olfactory cue. Animals experienced 15 trials with each drug reinforcer in a counterbalanced manner. Start latencies, run times, and retreat behaviors (a form of approach-avoidance conflict) provided behavioral indices of the subjects' motivation to seek the reinforcer on each trial. Responsiveness to each drug-paired cue was assessed after 7, 14, or 21 days of nonreinforced extinction trials. Other animals underwent conditioned place preference (CPP) testing to ensure that the two drug reinforcers were capable of producing drugcue associations.

Results While both drugs produced comparable CPPs, heroin served as a stronger incentive stimulus in the runway as evidenced by faster start and run times and fewer retreats. In contrast, cocaine- but not heroin-paired cues produced increases in drug-seeking behavior during subsequent extinction trials.
\end{abstract}

\footnotetext{
Z.-I. Su J J. Wenzel $\cdot$ R. Baird $\cdot$ A. Ettenberg $(\bowtie)$

Department of Psychology, University of California,

Santa Barbara, CA 93106-9660, USA

e-mail: ettenber@psych.ucsb.edu
}

Conclusions The subjects' responsiveness to drug-paired cues during extinction was not predicted by differences in the motivation to seek heroin versus cocaine during prior drug self-administration.

Keywords Cocaine $\cdot$ Heroin $\cdot$ Runway $\cdot$ Drug reinforcement · i.v. self-administration · Drug seeking · Motivation $\cdot$ Response reinstatement

\section{Introduction}

Drug relapse (i.e., the re-emergence of drug seeking after a protracted period of drug withdrawal) is among the most prominent characteristics of addiction (Mendelson and Mello 1996; O'Brien 1997). Relapse of drug-seeking behaviors can occur as a consequence of several factors including re-exposure to the drug itself (de Wit and Stewart 1983; Jaffe et al. 1989), exposure to other drugs of abuse (de Vries et al. 1999; de Wit and Stewart 1981, 1983), to stress (Ahmed and Koob 1997; Shaham et al. 1997), and/or to cues previously associated with the drug reinforcer (Carter and Tiffany 1999; Fuchs et al. 2004; Grimm et al. 2001; Kruzich et al. 1999). Of these various factors, the role of conditioned cues has received considerable attention in both the human and animal literature (e.g., see reviews by Crombag et al. 2008; Rohsenow et al. 1990). In humans, exposure to cues that have been previously associated with drug consumption can induce an intense subjective craving for the drug that is highly correlated with renewed drug abuse (Childress et al. 1998; Rohsenow et al. 1990; Withers et al. 1995). Similarly in animal models, reinstatement of operant responding for drug reinforcers can be induced by presentation of drug-paired contextual or discrete cues even after long periods of non-reinforced responding (extinction) 
or simply after the passage of time from previous drug exposure (e.g., Di Ciano and Everitt 2002; Kelamangalath and Wagner 2009; Lu et al. 2004; Shaham et al. 1997; Weiss et al. 2001).

Several aspects of the initial self-administration experience itself have been identified in animals as reliable predictors of subsequent renewed responding (including cue-induced reinstatement) after a period of drug withdrawal. These include the dose of the self-administered drug (Mantsch et al. 2001, 2004), the length of the training period (Deroche et al. 1999), and the initial schedule of reinforcement (Acosta et al. 2008; Valles et al. 2006). For example, Ahmed and Koob (1998) have shown that animals permitted $6 \mathrm{~h}$ of daily access to intravenous (i.v.) cocaine demonstrate escalating rates of self-administration over days (compared to animals provided $1 \mathrm{~h}$ of daily cocaine access) and are more responsive to factors that induce a reinstatement of drug seeking following periods of drug withdrawal (Ahmed and Cador 2006; Kippin et al. 2006; Knackstedt and Kalivas 2007). Thus, animals exhibiting higher rates of drug self-administration appear to be more likely to re-engage drug seeking after the drug reinforcer has been removed. However, it remains unknown whether or not such conclusions generalize to comparisons between drug reinforcers.

To address this question, the authors have employed an operant runway model that assesses a subject's motivation to seek goal-box incentive stimuli, including drugs of abuse (see review by Ettenberg 2009). In this model, the time required to run a straight alley and enter a goal box for an i.v. injection of a drug reinforcer serves as an index of the subjects' motivation to seek the drug reinforcer in question. In previous work, i.v. heroin and cocaine have been shown to produce highly distinct patterns of runway behavior. When heroin serves as the reinforcer, animals initiate trials quickly, run fast, and proceed directly into the goal box. In contrast, rats running for cocaine, while initiating trials quickly, develop a distinct pattern of approach-avoidance conflict about goal box entry that gets progressively stronger over trials (e.g., Ettenberg and Geist 1993). The conflict is operationally quantified by the number of "retreat behaviors" that occur, i.e., the number of times an animal runs quickly to the threshold of the goal but then stops, turns, and retreats back toward the start box. Such behavior is prototypical of animals approaching a goal box with which they have concurrent positive and negative associations such as a goal box where both food + shock are delivered upon entry (Cohen et al. 2009; Geist and Ettenberg 1997; Miller 1944). Indeed, cocaine administration is now wellknown to have dual and opposing affective consequences where the initial rewarding/euphoric state is followed by a "crash" associated with anxiety, anhedonia, restlessness, and cravings (e.g., Childress et al. 1988; Van Dyke and
Byck 1982; see review by Ettenberg 2004). The slow development of cocaine-induced retreat behaviors over several trials is thought to stem from this delay in the onset of the drug's aversive effects and the consequent need for additional runway drug pairings in order for the animal to adequately form the association between such effects and the goal box (Ettenberg 2009). In any event, the runway data clearly suggest that the motivation of subjects to seek heroin and cocaine are qualitatively different from one another, with heroin having more purely positive behavioral effects (approach) and cocaine having mixed positive and negative effects (approach and avoidance). It was of interest, therefore, to test the hypothesis that such differences during self-administration would extend to differences in the rats' responsiveness to a cocaine- or heroin-paired cue after varying periods of non-reinforced trials (i.e., extinction).

\section{Materials and methods}

\section{Subjects}

The subjects were male Sprague-Dawley rats $(n=50)$ weighing $330-360 \mathrm{~g}$ at the time of surgery (Charles River Laboratories, Wilmington, MA, USA). Rats were individually housed in plastic cages within a temperaturecontrolled $\left(23^{\circ} \mathrm{C}\right)$ vivarium maintained on a reverse 12 $\mathrm{h}$ light-dark cycle (lights off at 0800 hours). Free access to food (Purina Rat Chow) and water was provided throughout the duration of the study. All animal handling and experimental procedures adhered to the NIH Guide for the Care and Use of Laboratory Animals and were reviewed and approved by the University of California at Santa Barbara's Institutional Animal Care and Use Committee.

\section{Surgery}

Rats were acclimated to human handling for 1 week prior to i.v. catheterization. Each rat was then deeply anesthetized via isoflurane inhalation (4\% for induction and $1.5-2.5 \%$ for maintenance) and fitted with a catheter $(13 \mathrm{~mm}$ of polyethylene tubing, $0.3 \mathrm{~mm}$ inner and $0.64 \mathrm{~mm}$ outer diameters; Dow Corning Corp, Midland, MI, USA) that was inserted into the right jugular vein and secured by silk sutures. The open end of the catheter was passed subcutaneously to a 2-mm hole located on the midline of the animal's back. This end of the catheter has been preassembled to fit into a stainless steel guide cannula (Item 313G; Plastics One, Roanoke, VA, USA) that was in turn affixed with dental cement to a 2-cm square of Mersilene surgical mesh (Bard; Warwick, RI, USA). The surgical mesh was laid flat on the animal's back and secured by 
suture to the subdermal tissue and the guide cannula protruded through the hole perpendicular to the surface of the animal's back. During surgery, rats were treated with atropine $(0.02 \mathrm{mg} / \mathrm{kg}$, intramuscularly) to prevent respiratory congestion and the non-opiate analgesic flunixin meglumine (Phoenix Pharmaceuticals, Belmont, CA, USA; $1.3 \mathrm{mg} / \mathrm{kg}$, subcutaneously) to reduce post-surgical pain. Following surgery, rats received the antibiotic ticarcillin disodium/clavulanate potassium (Timetin; $50 \mathrm{mg} / \mathrm{kg}$, i.v. $)$ and $0.1 \mathrm{ml}$ of heparin $(6.0 \mathrm{IU} / 0.1 \mathrm{ml}$ prepared in $0.9 \%$ physiological saline, i.v.) as a prophylactic measure against microbial infection and to promote catheter patency.

One week was provided for subjects to recover from surgery during which the catheters were flushed daily with $0.1 \mathrm{ml}$ of antibiotic (ticarcillin disodium/clavulanate potassium, $20 \mathrm{mg} / \mathrm{kg}$, i.v.) and $0.1 \mathrm{ml}$ of heparinized $0.9 \%$ physiological saline to maintain catheter patency. In addition, rats received $3.0 \mathrm{ml}$ of $0.9 \%$ physiological saline subcutaneously once each day for three consecutive days post-surgery to prevent dehydration. Topical antibiotics bacitracin, neomycin, and polymyxin B (Neosporin, Pfizer, New York, NY, USA) were applied to all incisions to aid healing and prevent infection. Once each week, catheter patency was confirmed by observing the behavioral impact of an i.v. injection of the fast-acting barbiturate, methohexital sodium (Brevital; $2.0 \mathrm{mg} / \mathrm{kg} / 0.1 \mathrm{ml}$ ). Animals that did not lose their righting reflex in response to this challenge were re-implanted with a new catheter using the left jugular vein and given additional days for recovery.

\section{Drugs}

Both the cocaine hydrochloride (cocaine, $1.0 \mathrm{mg} / \mathrm{kg}$ ) and diacetylmorphine (heroin, $0.1 \mathrm{mg} / \mathrm{kg}$ ) were provided by the National Institute of Drug Abuse. Each drug was dissolved in a vehicle of $0.9 \%$ physiological saline and delivered in a volume of $0.1 \mathrm{ml}$ over a period of $4.3 \mathrm{~s}$ via a $10-\mathrm{ml}$ syringe that was seated in a motorized syringe pump (Razel Scientific Instruments, St. Albans, Vermont, USA). The doses selected for study had been shown to be maximally effective (produced the fastest start times, run times, and fewest retreats) in prior runway studies (e.g., Ettenberg et al. 1996; Ettenberg and Geist 1993; Guzman and Ettenberg 2004; McFarland and Ettenberg 1997; Raven et al. 2000).

\section{Runway apparatus}

All runway testing was conducted in two straight-arm alleys $(160 \mathrm{~cm}$ in length $\times 12 \mathrm{~cm}$ wide $\times 44 \mathrm{~cm}$ high). A start box and goal box (each $23 \times 20 \times 44 \mathrm{~cm}$ ) were attached to opposite ends of each runway. The floor of each apparatus consisted of $3 \mathrm{~cm}$ steel rods laid in parallel $1.2 \mathrm{~cm}$ apart perpendicular to the runway walls. Embedded in the side walls of each runway were 13 evenly spaced pairs of infrared photoemitters (on one side wall) with corresponding infrared detectors on the opposite wall. The first pair was located within the start box and the 13th pair inside the goal box. The output from these sensors was fed to a desktop computer via a custom Any-Maze interface (AMi; Stoetling Co, Wood Dale, IL, USA) that recorded the precise location of the animal in the alley in real time throughout the course of each trial.

Suspended above each runway were two long magnetic rails (spaced $3 \mathrm{~cm}$ apart) that ran in parallel along the entire length of the apparatus. Positioned between the rails was a liquid swivel (375-22PS, Instech Labs Inc.) that connected the guide cannula on the animal's back to the $10-\mathrm{ml}$ drugfilled syringe, via PE-20 tubing. A flat plastic disk was secured to the midsection of each swivel to prevent it from falling through the gap between the magnetic rails. A pot magnet was attached to the underside of the disk with the polarity arranged to repel the charge of the magnetic rails. The resulting magnetic repulsion between the swivel assembly and the rails permitted the swivel to float slightly above the tracks. Thus, this system served as a low-friction mechanism that allowed the rat to move freely throughout the alley, pulling the swivel assembly along behind and above it as it moved (for more details, see Geist and Ettenberg 1990).

\section{Runway self-administration}

Rats were habituated to the apparatus during a single 10min trial during which they were permitted to explore the runway and start box (but not the goal box). On the following day, each animal was individually connected to the drug delivery system and placed into the start box where, after $5 \mathrm{~s}$, the start door opened and the trial commenced. Upon the rat's entry into the goal box, the goal door was closed (to prevent re-tracing) and the syringe pump activated to deliver the drug reinforcer. Rats were left in the goal box for an additional 5-min and then returned to their home cages.

Prior to each runway trial, an olfactory cue was provided (McCormick's pure orange or almond food extract; Sparks, MD, USA) by placing cotton swabs infused with $3 \mathrm{ml}$ of the scented extract under the start box, halfway down the runway, and under the goal box. A fan was used to continuously aerate the runways with the odor. Animals experienced 15 single-daily runway training trials for either heroin or cocaine reinforcement in the presence of a single distinct olfactory cue (either almond or orange scents). Beginning on the trial/day 16 , the drug reinforcer was switched to the alternative drug and 15 additional daily trials were conducted in the presence of the alternate scent. 
Half of the animals experienced heroin trials first and then cocaine (and vice versa), and the two scents were also counterbalanced across groups. Following the completion of this 30-day runway self-administration phase, rats underwent either 6,13 , or 20 days of non-reinforced, noncued extinction trials followed by a single test trial in which runway responding was assessed upon the presentation of either the cocaine- or heroin-paired cue.

Three dependent measures were collected on every trial: start latency (time from opening start door to a break in photobeam \#2 located just outside of the start box), run time (the time required to traverse the runway and enter the goal box, i.e., the time elapsed between breaking photocell \#2 outside the start box and \#12 just inside the goal box), and retreat frequency (the number of times the animal ceased its forward locomotion toward the goal, stopped, and reversed its direction back toward the start box; the criterion for a retreat was a reversal in direction for a distance spanning a minimum of two photocell breaks).

\section{Conditioned Place Preference apparatus}

A conditioned place preference (CPP) test was conducted to ensure that the doses of the drug reinforcers used in the runway were sufficiently salient to produce drug-place associations after daily 5-min pairings. Two identical CPP apparatus consisted of a wood-constructed rectangular enclosures $(156 \mathrm{~cm}$ long $\times 34 \mathrm{~cm}$ wide $\times 30 \mathrm{~cm}$ high $)$ that could be sub-divided into three separate compartments: Two larger chambers $(61 \times 30 \mathrm{~cm})$, one painted black and the other white, were separated by a smaller gray intermediate chamber $(34 \times 30 \mathrm{~cm})$. The black side of the apparatus contained acrylic (Plexiglas ${ }^{\circledR}$ ) flooring, the floor of the white compartment was covered with soft wood bedding, and the gray middle section had a painted wood surface floor. The black compartment was scented with a dilute $2 \%$ acetic acid solution (a single wipe along each wall $5 \mathrm{~cm}$ from the floor); the white compartment received no additional odor cues. Thus, each CPP apparatus provided three distinct environments that differed in color, texture, and scent. We have found these procedures to yield two conditioning environments (the white and dark compartments) for which rats exhibit no reliable inherent preferences prior to conditioning. During preference tests, the dividing walls were removed to allow the animal full access to each of the three compartments of the apparatus. Situated above each apparatus was a digital camera that detected and recorded the precise location of the animal in real time via a desktop computer running Any-Maze software (Stoetling Co, Wood Dale, IL, USA).

The CPP procedure consisted of a 10-day protocol that involved a one-trial pre-conditioning preference test, eight place conditioning trials, and a final post-conditioning preference test. On day 1 , the interior walls separating each compartment were removed, each rats was placed into the middle gray section of the apparatus, and the time spent in each of the three compartments was recorded over a 15 -min "baseline". This was done to ensure that no animal had strong initial side preferences prior to drug-place conditioning. Each rat then completed 8 days of conditioning: On the first such day, each animal received an i.v. injection of either vehicle or drug and was then placed into either the white or black compartment for 5 min (thereby modeling the temporal parameters of the drug-goal box associations in the runway). On the following day, each rat received the alternate treatment (either drug or vehicle) and was placed in the alternate colored environment. This continued in an alternating manner until each subject had experienced eight conditioning trials. One group of animals experienced the effects of i.v. cocaine $(1.0 \mathrm{mg} / \mathrm{kg})$ in one side and i.v. saline in the other, a second group experienced i.v heroin $(0.1 \mathrm{mg} / \mathrm{kg})$ in one side and saline in the other, and a final group experienced cocaine in one compartment and heroin in the other. The order in which subjects received the drug or vehicle, as well as the side of the apparatus paired with a given treatment, was counterbalanced across all animals and between groups (i.e., an unbiased CPP design was employed; see Carr et al. 1988). The day after the final conditioning trial, each subject underwent a 15 -min CPP test precisely as described for the baseline session. CPPs were determined by comparing the time spent in the drug-paired environment with that spent in the vehicle-paired environment or, in the case of cocaine versus heroin, in one drug-paired environment compared to the other.

\section{Results}

\section{Runway self-administration}

Animals traversed the alley once a day over 15 days for either single cocaine $(n=24)$ or heroin $(n=26)$ i.v. infusions followed by 15 additional days/trials for the alternate drug reinforcer. Figure 1 depicts the runway behavior of these subjects expressed as 3-day average scores (to smooth the variability inherent of single daily runway measures). A mixed twofactor (Group $\times$ Trial) analysis of variance (ANOVA) computed on the start latency (Fig. 1a) yielded a significant Group $\times$ Trial interaction $(F(9,48)=4.291, p<0.01)$, but no main effects for Trial or Group $(p>.05)$. Thus, changes in start latencies differed across groups as trials progressed. Indeed, while both groups improved their start times with repeated testing, cocaine-reinforced animals started much more slowly than heroin-reinforced animals during the early trials but initiated responding with increasing speed (shorter latencies) as testing continued. An independent $t$ test conducted on very 



Fig. 1 Mean $( \pm$ SEM) starts latency (a), run time (b), and retreats (c) for animals running an alley for i.v. heroin or cocaine. Data were averaged across 3 days/trials. Animals ran exclusively for one drug reinforcer for 15 trials and then were switched to the alternate reinforcer for an additional 15 trials (shift denoted by the dotted vertical line)

first day of runway training revealed no significant differences between the heroin- or cocaine-reinforced animals $(p>0.05)$, suggesting that the initial disparity between the two groups seen on the first data point (averaged across trials 1-3) is attributable to performance on trials 2 and 3 (i.e., only after the rats had experienced the drugs in the goal box). Once the drug reinforcers were transposed, rats shifting from cocaine to heroin showed an immediate and reliable decrease in start latencies while animals shifting from heroin to cocaine increased their start latencies. Thus, the failure to observe any overall main effect for Trial or Group is not surprising since the reversal in group performance after the drug reinforcers were switched essentially offset the differences observed prior to the switch.

Mean Group run times ( \pm SEM) are depicted in Fig. 1b. Here, the mixed two-factor (Group $\times$ Trial) ANOVA yielded a significant main effect for Trial $(F(9,48)=2.341, p<0.05)$ and Group $(F(1,48)=16.399, p<0.01)$ as well as a Group $\times$ Trial interaction $(F(9,48)=6.692, p<0.01)$. Heroin- and cocaine-reinforced subjects initially traversed the runway at comparable speeds (trials 1-3), but over time the cocainereinforced rats took progressively longer to enter the goal box while heroin-reinforced animals tended to maintain or reduce their run times. When the reinforcers were transposed, animals moving from heroin to cocaine increased their run times (i.e., ran more slowly) while animals moving from cocaine to heroin maintained or continued to reduce their run times.

Rats working for i.v. cocaine developed a conflict about goal box entry - as evidenced by a progressive increase in retreat behaviors over trials - an effect not observed in animals working for heroin (Fig. 1c, left panel). A mixed two-factor ANOVA (Group $\times$ Trial) yielded significant main effect for Trials $(F(9,48)=27.513, p<0.01)$ and a significant Group $\times$ Trial interaction $(F(9,48)=13.810$, $p<0.01)$, but no main effect of Group $(p>0.05)$. Both groups of animals exhibited few and comparable numbers of retreats during initial trials of testing, and only the cocaine-reinforced rats exhibited a progressive increase in retreat frequency as testing continued. After the two drug reinforcers were transposed, those rats transitioning from heroin to cocaine exhibited a progressive increase in retreat frequency similar to that observed in those animals that ran for cocaine during the first half of the drug selfadministration session. In animals transitioning from cocaine to heroin, no additional increase in retreat frequency was observed. Of course, when averaged across groups, retreat frequency tended to increase over trials (Trial effect) with the effect due primarily to the elevation in retreats observed in the heroin to cocaine subjects (Group $\times$ Trial interaction).

\section{Runway cue-induced reinstatement}

The responsiveness of subjects to the cocaine- or heroinpaired olfactory cues was assessed on the 7 th, 14 th, or 21 st day of extinction. A comparison of the mean test day performance of animals that transitioned from cocaine to heroin versus those that transitioned from heroin to cocaine identified no group differences for start latencies, run times, or retreats (Bonferroni-protected independent sample $t$ tests). Thus, the rats' behavioral response to the cues on test day 
occurred in a comparable manner independent of initial drug order during self-administration. The data for these two subgroups were therefore combined and averaged for each drug-cue condition (i.e., animals presented with the cocaine-paired scent were compared to the animals presented with the heroin-paired scent independent of the initial order of drug exposure).

Separate mixed two-factor (Group $\times$ Trial) ANOVAs were employed to analyze the behavioral effects of presenting subjects with the heroin or cocaine cue at different times after drug self-administration had terminated. The impact of the cocaine-paired cue was assessed by separate ANOVAs on start latencies, run times, and retreats. The same was the case for the subjects presented with the heroin cue. For each ANOVA, there was a single independent factor (Group; i.e., different animals were tested at different withdrawal periods; 7, 14, or 21 days of extinction) and a single repeated measures factor (Trial) where cue-induced performance on test day was compared to the mean baseline performance over the prior five extinction trials.

The ANOVAs on start latency data did not yield any significant main effects or interactions for either the heroin or cocaine cue $(p>0.05)$. Thus, neither the number of days of withdrawal nor the presentation of a drug-paired cue altered the animals' time to initiate responding relative to baseline/extinction performance. In contrast, statistically reliable effects were identified for both run times and retreats (Figs 2 and 3, respectively).

For animals presented during extinction with the cocaine cue (Fig. 2, left side), there was a statistically reliable main effect of Trial $(F(1,23)=4.786, p=0.04)$-animals ran

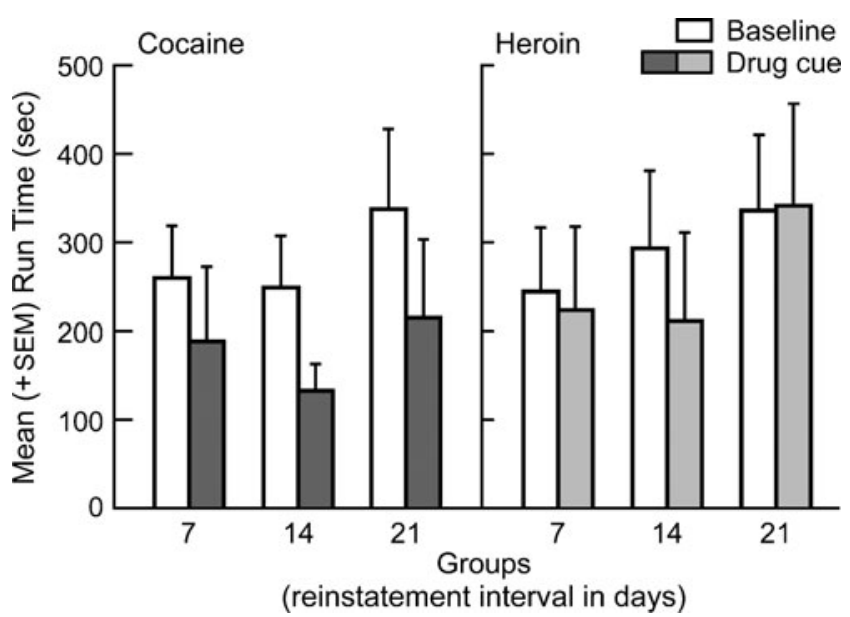

Fig. 2 Mean (+SEM) run times upon presentation of a cocaine- or heroin-paired olfactory cue presented to different groups of animals after 7, 14, or 21 days of non-reinforced extinction trials. Cue-induced responding (shaded bars) on test day are compared to an extinction baseline (open bars) computed as the average of the five preceding extinction trials

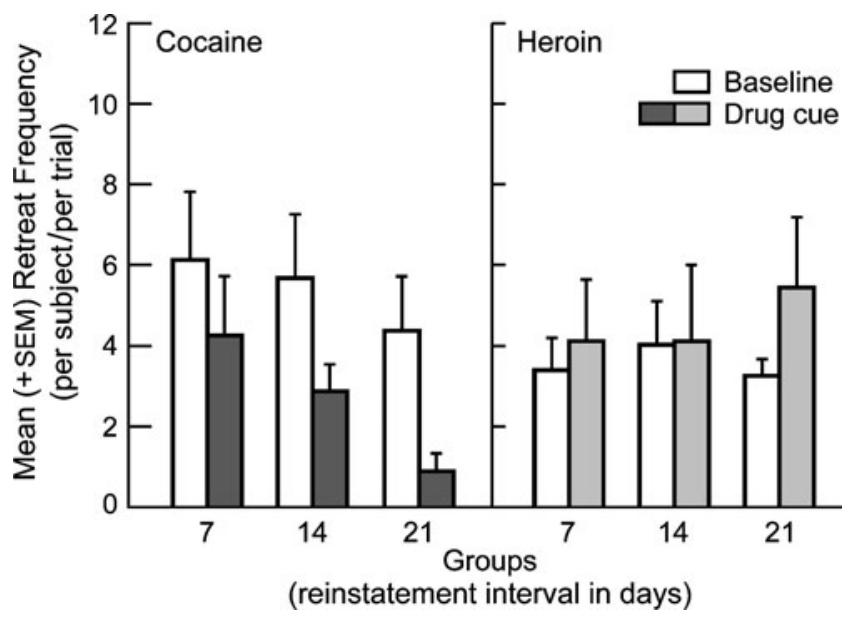

Fig. 3 Mean (+SEM) retreat frequency on test day (shaded bars) compared to baseline (open bars) of different groups of animals exposed to a cocaine- or heroin-paired cue after 7, 14, or 21 days of extinction

faster (exhibited shorter run times) in the presence of the cocaine-paired cue than they did during prior non-cued extinction trials. There was no main effect of Group nor a Group $\times$ Trial interaction. The comparable ANOVA computed on the run time data for animals presented with the heroin cue (Fig. 2, right side) yielded no statistically significance results. For retreat frequency (Fig. 3), the ANOVAs yielded results comparable to those computed on run times. For the cocaine-cued animals, (Fig. 3, left side), retreats were significantly reduced in the presence of the cue (test day) compared to non-cued extinction trials (i.e., there was a strong main effect of Trial: $F(1,23)=10.496$, $p=0.004)$. There was again no effect of Group, and while the Group $\times$ Trial interaction was marginal $(p<.07)$, it failed to reach statistical significance. As was the case for run times, the ANOVA computed on the data from the heroincued animals (Fig. 3, right side) revealed no statistically reliable effects $(p>0.05)$.

\section{Conditioned place preference}

An analysis of preconditioning baseline data confirmed that, in all three groups, there were no inherent preferences for one side of the apparatus relative to the other (Bonferroni-corrected two-tailed repeated-measures $t$ tests). After place conditioning, both of the reinforcers that were employed in the prior runway study $(1.0 \mathrm{mg} / \mathrm{kg}$ cocaine and $0.1 \mathrm{mg} / \mathrm{kg}$ heroin) produced statistically reliable increases (assessed by Bonferroni-corrected $t$ tests) in time spent in the drug-paired environment compared to that in the vehicle-paired environment: cocaine $(t(9)=3.480, p<0.01)$ and heroin $(t(11)=4.666, p<0.001)$. When the two reinforcers were directly compared against each other within the same animal, there was a slight, but non-significant 
preference for the heroin-paired over the cocaine-paired compartment $(t(12)=1.2, p>0.05)$. These results are depicted in Fig. 4 and clearly demonstrate that the doses of cocaine and heroin employed here were sufficiently salient to produce reliable drug-place associations even after only four 5-min drug-place pairings and that the initial impact of the drugs (during the first $5 \mathrm{~min}$ post-injection) was comparably rewarding for both compounds.

\section{Discussion}

The current data confirm and extend previous findings from our laboratory that the behavior of animals working for i.v. cocaine qualitatively differs from that of animals working for i.v. heroin (e.g., Ettenberg and Geist 1993). In the initial portion of self-administration testing, heroin-reinforced animals consistently produced faster start latencies and run times and exhibited little or no ambivalence about goalbox entry (i.e., very few retreats). In contrast, animals running for i.v. cocaine took comparatively longer to initiate runway responding (elevated start latencies) and longer to enter the goal box (elevated run times). The elevation in the run times of the cocaine-reinforced rats is likely due, in large part, to the progressive development of retreat behaviors since, to state the obvious, animals running back and forth in the alley will take longer to enter the goal box than animals that do not exhibit such behaviors. These retreats are thought to represent a form of approach-avoidance conflict behavior that stems from an ambivalence about entering a goal box with which the

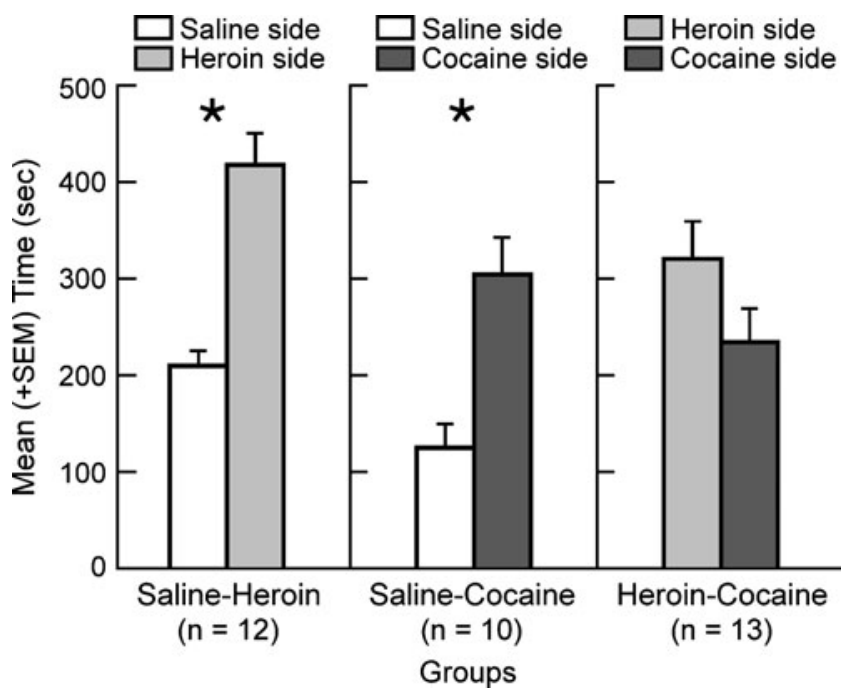

Fig. 4 Mean (+SEM) test days performance during a 5-min CPP test. Data reflect the time spent on the heroin-paired versus saline-paired side of a CPP apparatus during preference testing (left), on the cocaine-paired versus saline-paired side of the CPP apparatus (middle), and on the cocaine- versus heroin-paired sides of the preference apparatus (right). ${ }^{*} p<0.01$ (saline versus drug compartment) subject has mixed positive and negative associations (see reviews by Ettenberg 2004, 2009). Thus, for example, the behavior of cocaine-reinforced rats in the runway is comparable to that exhibited by animals approaching a goal box whose entry has clearly discernible positive and negative consequences (e.g., where both food and footshock are delivered; Cohen et al. 2009; Geist and Ettenberg 1997; see also Miller 1944), and like other forms of conflict, retreat behaviors are dose-dependently reduced by pretreatment with compounds having known anxiolytic effects such as diazepam (Ettenberg and Geist 1991), busiprone, (Ettenberg and Bernardi 2006), or alcohol (Knackstedt and Ettenberg 2005). When CPP procedures are used, animals develop preferences for places paired with the immediate effects of i.v. cocaine, but aversions for environments paired with the effects present $15 \mathrm{~min}$ postinjection (e.g., Ettenberg et al. 1999; Ettenberg and Bernardi 2007; Knackstedt et al. 2002). Collectively, such findings suggest that acute administration of the drug has immediate positive (rewarding) and delayed negative (anxiogenic) consequences, a conclusion substantiated by subjective reports of human cocaine users (van Dyke and Byck 1982; Nelson et al. 2005; Lundahl and Lukas 2007).

A novel aspect of the current study was the examination of the behavioral response of animals that were shifted from heroin to cocaine and vice versa. This permitted the determination of how discernable each reinforcer was from one another by examining whether or not and how quickly the animals would alter their pattern of runway behavior once the drug shift was made. The results clearly demonstrate that when rats are transitioned from one drug reinforcer to another, their runway responding quickly adopts the pattern associated with the "new" reinforcer (see Fig. 1). Thus, when animals were shifted from heroin to cocaine, they immediately exhibited slower start latencies, slower run times, and a progressive increase in retreat behaviors. The impact of the shift from cocaine to heroin was more complex since the animals had already established mixed positive and negative associations with the goal box prior to the reinforcer shift. Hence, one would expect such associations to have a continued impact even after the shift to heroin reinforcement. We know from previous work that retreat behaviors normally continue to rise during the first 3 weeks of daily testing and that an intervention that alters the negative impact of cocaine after retreats have already developed (e.g., the addition of heroin to the cocaine solution) truncates the further increase in retreats that would otherwise have occurred (Guzman and Ettenberg 2004). Similarly, in the current study, when animals were shifted from cocaine to heroin, a continued rise of retreats and further slowing of run times did not materialize and both measures remained stable or slightly improved. It therefore seems clear that rats readily 
discriminate between the impact of heroin and cocaine and adjust their approach behavior accordingly. If run times reflect the undrugged subjects' motivation to seek the goal box reinforcer each day (as numerous studies over many decades have suggested; see review by Ettenberg 2009), then the distinctive patterns of responding in animals running for cocaine versus heroin suggest that the underlying positive incentive properties of the drug (i.e., those aspects of a stimulus that induce an animal to approach) may be stronger for heroin than they are for cocaine.

Such a conclusion is consistent with reports that the administration of the opiate drug, buprenorphine, can reduce cocaine self-administration in rats (Comer et al. 1996; Winger et al. 1992) and decrease preferences for cocaine in human addicts (Foltin and Fischman 1994, 1996). However, it has also been shown that animals given a direct choice between responding for cocaine (on one lever) or heroin (on an alternate lever) demonstrate strong preferences for the cocaine (Ward et al. 2005). Of course the current runway procedure does not offer animals a direct choice between the two drugs (a choice that is very likely affected by differences in the pharmacokinetics of cocaine and heroin). So while the initial positive/rewarding effects of cocaine might be stronger than those of heroin, thereby producing preferences for cocaine over heroin in a two-lever choice situation, the current runway data suggest that when the animal is tested in a non-drugged state, its motivation to seek heroin (as indicated by all three behavioral runway measures) appears to be more positive (or less mixed) than that for cocaine.

The authors recognize that the observed differences in the response patterns of heroin- and cocaine-reinforced runway behavior may stem in part from the particular doses that were selected for study. Of course, as noted above, these doses were selected because we have in the past found them to produce the fastest start and run times and the fewest retreats for each drug (see "Materials and methods" for reference citations). However, the issue being addressed here is not whether or not the particular doses employed here generalize to other heroin and cocaine doses but rather whether the apparent enhanced motivation to seek one drug over another (at any dose) during selfadministration, serves as a reliable predictor of the animals' later responsiveness to cues paired with each of those drugs following periods of non-reinforced extinction responding. As discussed in the "Introduction" of this paper, several reports have shown that the pattern of responding during self-administration can serve as a predictor of the subjects' vulnerability to factors that induce a reinstatement of drugseeking following a period of drug withdrawal (e.g., Ahmed and Cador 2006; Kippin et al. 2006; Knackstedt and Kalivas 2007). A novel finding in the current study is that despite what appears to be a stronger motivation to seek heroin versus cocaine during self-administration, the cocaine cue was more effective at potentiating responding during extinction than was the heroin-paired cue. Once again it might be suggested that heroin dose in the current study was less salient than the cocaine dose (due to heroin's slower onset and longer duration of action), and hence, the strength of the resulting association between the external cue and the drug was weaker for heroin that it was for cocaine. This explanation, however, seems unlikely for two reasons: First, the almost immediate shift in the pattern of responding that occurred when animals were transitioned to or from heroin suggests that its presence was clearly discernible by the animals, and second, the demonstration that the same heroin dose employed in the runway was effective at producing significant conditioned place preferences (even after only four drug-place pairings) suggests that the animals were capable of forming associations between external cues and the dose of the heroin reinforcer used in the current study. A more parsimonious explanation for the present results is that the pattern and strength of responding during self-administration may not always predict a subject's responsiveness to those drug-paired cues that have been shown to induce a reinstatement of drug-seeking behavior after a period of non-reinforced responding.

One might question why, if heroin is in fact a more potent incentive stimulus than cocaine at the doses employed here, did it not produce a stronger CPP when directly compared to cocaine (Fig. 4, right panel)? This may simply be a consequence of the fact that four drug-place pairings over 8 days was insufficient for such differences to emerge. Indeed, even in the runway, the conflict behavior (retreats) in cocaine animals do not develop until at least 9 days of consecutive testing. We would therefore predict that a more prolonged CPP conditioning protocol would ultimately reveal the differences between heroin and cocaine that the runway has identified in this and prior studies.

Several aspects of the current experimental protocol differ from those typically employed in animal studies of cue-induced reinstatement of drug-seeking behavior. Most prominent among these is that fact that the rats in the current study drug self-administration involved single daily exposure to the drug reinforcer over a 30-day period. In typical response-reinstatement studies, the initial lever-press self-administration experience results in numerous drug infusions per day (for a review of reinstatement methodologies, see Shaham et al. 2003). As a result, although run times slowed for all groups over the course of extinction (see Fig. 2), the effect was modest and not statistically significant. This may account for why there was no reliable response potentiation observed upon presentation of the heroin-paired cue - a finding that contrasts with the results of other studies that employed traditional lever-press self- 
administration methods (e.g., Bossert et al. 2005; de Wit and Stewart 1983; Leri and Stewart 2001; Shalev et al. 2001; Zhou et al. 2009). It should be noted that reliable response reinstatement of heroin seeking has been reported using the runway model (e.g., Ettenberg and Geist 1993; McFarland and Ettenberg 1997), but these studies included more exposure to heroin during training and/or longer periods of withdrawal to ensure a significant weakening in run times prior to the reinstatement challenge. The current findings are important in that they show that in animals experiencing only modest daily drug exposure (which undoubtedly has relevance for a considerable subset of the human drug-using population), cocaine-paired cues have a stronger impact on potentiating drug-seeking behavior than do heroin-paired cues. This suggests that the development of a more pernicious form of drug use likely has its seeds in early "recreational" use of the drug in question and that the "risk" of abuse may be greater for cocaine than for heroin.

A final observation worth noting is what appears to be a time-dependent reduction in retreat frequency (in the cocaine-cue condition) as extinction progressed (Fig. 3, left panel). Although the Group $\times$ Trial interaction on those data was only marginal $(p<.07)$, the pattern of behavior observed seems to mirror the "incubation of cocaine craving" phenomenon in which the effectiveness of drugpaired cues to reinstate drug-seeking increases as the period of drug withdrawal is extended (e.g., Ahmed and Cador 2006; Grimm et al. 2001; Lu et al. 2003, 2004; Shalev et al. 2001; Tran-Nguyen et al. 1998; Zhou et al. 2009). The current results suggest the intriguing possibility that the incubation phenomenon may stem, at least in part, from a differential rate at which the negative relative to the positive associations that animals have established between cocaine and cocaine-paired cues weaken over time.

Acknowledgments The authors acknowledge, with gratitude, Stephanie Waldroup and Gleb Kichaev for their assistance in various aspects of the project and Dr. Osnat Ben-Shahar for her input on the design and conception of these studies. This research was funded by NIDA grant DA05041 awarded to A.E.

Open Access This article is distributed under the terms of the Creative Commons Attribution Noncommercial License which permits any noncommercial use, distribution, and reproduction in any medium, provided the original author(s) and source are credited.

\section{References}

Acosta JI, Thiel KJ, Sanabria F, Browning JR, Neisenwander JL (2008) Effects of schedule of reinforcement on cue-elicited reinstatement of cocaine-seeking behavior. Behav Pharmacol 19:129-136

Ahmed SH, Cador M (2006) Dissociation of psychomotor sensitization from compulsive cocaine consumption. Neuropsychopharmacology 31:563-571
Ahmed SH, Koob GF (1997) Cocaine but not food-seeking behavior is reinstated by stress after extinction. Psychopharmacology 132:289-295

Ahmed SH, Koob GF (1998) Transition from moderate to excessive drug intake: change in hedonic set point. Science 282:298-300

Bossert JM, Ghitza UE, Lu L, Epstein DH, Shaham Y (2005) Neurobiology of relapse to heroin and cocaine seeking: an update and clinical implications. Eur J Pharmacol 526:36-50

Carr GD, Phillips AG, Fibiger HC (1988) Independence of amphetamine reward for locomotor stimulation demonstrated by conditioned place preference. Psychopharmacology 94:221-226

Carter BL, Tiffany ST (1999) Meta-analysis of cue-reactivity in addiction research. Addiction 94:324-340

Childress A, Ehrman R, McLellan AT, O'Brien C (1988) Conditioned craving and arousal in cocaine addiction: a preliminary report. NIDA Res Monogr 81:74-80

Childress AR, McLellan AT, Ehrman R, O’Brien CP (1998) Classically conditioned responses in opioid and cocaine dependence: a role in relapse? NIDA Res Monogr 84:25-43

Cohen A, Young R, Velazquez M, Noorbehesht K, Groysman M, Ben-Shahar OM, Ettenberg A (2009) Anxiolytic effects of nicotine in a rodent test of approach-avoidance conflict. Psychopharmacology 204:541-549

Comer SD, Lac ST, Wyvell CL, Carroll ME (1996) Combined effects of buprenorphine and a nondrug alternative reinforcer on IV cocaine self-administration in rats maintained under FR schedules. Psychopharmacology 124:355-360

Crombag HS, Bossert JM, Koya E, Shaham Y (2008) Context-induced relapse to drug seeking: a review. Philos Trans R Soc Lond B Biol Sci 363:3233-3243, Review

Deroche V, Le Moal M, Piazza PV (1999) Cocaine self-administration increases the incentive motivational properties of the drug in rats. Eur J Neurosci 11:2731-2736

De Vries TJ, Schoffelmeer AN, Binnekade R, Vanderschuren LJ (1999) Dopaminergic mechanisms mediating the incentive to seek cocaine and heroin following long-term withdrawal of IV drug self-administration. Psychopharmacology 143:254260

de Wit H, Stewart J (1981) Reinstatement of cocaine-reinforced responding in the rat. Psychopharmacology 75:134-143

de Wit H, Stewart J (1983) Drug reinstatement of heroin-reinforced responding in the rats. Psychopharmacology 79:29-31

Di Ciano P, Everitt BJ (2002) Reinstatement and spontaneous recovery of cocaine-seeking following extinction and difference durations of withdrawal. Behav Pharmacol 13:397-405

Ettenberg A (2004) Opponent processes properties of selfadministered cocaine. Neurosci Biobehav Rev 27:721-728

Ettenberg A (2009) The runway model of drug self-administration. Pharmacol Biochem Behav 91:271-277

Ettenberg A, Bernardi RE (2006) Anxiolytic actions of busiprone in a runway model of intravenous cocaine self-administration. Pharmacol Biochem Behav 2:393-399

Ettenberg A, Bernardi RE (2007) Effects of busiprone on the immediate positive and delayed negative properties of intravenous cocaine as measured in the conditioned place preference test. Pharmacol Biochem Behav 87:171-178

Ettenberg A, Geist TD (1991) An animal model for investigating the anxiogenic effects of self-administered cocaine. Psychopharmacology 103:455-461

Ettenberg A, Geist TD (1993) Qualitative and quantitative differences in the operant runway behavior of rats working for cocaine and heroin reinforcement. Pharmacol Biochem Behav 44:191-196

Ettenberg A, MacConell LA, Geist TD (1996) Effects of haloperidol in a response-reinstatement model of heroin relapse. Psychopharmacology 124:205-210 
Ettenberg A, Raven MA, Danluck DA, Necessary BD (1999) Evidence for opponent-process actions of intravenous cocaine. Pharmacol Biochem Behav 64:507-512

Foltin RW, Fischman MW (1994) Effects of buprenorphine on the self-administration of cocaine by humans. Behav Pharmacol 5:78-89

Foltin RW, Fischman MW (1996) Effects of methadone or buprenorphine maintenance on the subjective and reinforcing effects of intravenous cocaine in humans. J Pharmacol Exp Ther 278:11531164

Fuchs RA, Evans KA, Parker MP, See RE (2004) Differential involvement of orbitofrontal cortex subregions in conditioned cue-induced and cocaine-primed reinstatement of cocaine seeking in rats. J Neurosci 24:6600-6610

Geist TD, Ettenberg A (1990) A simple method for studying intravenous drug reinforcement in the runway. Pharmacol Biochem Behav 36:703-706

Geist TD, Ettenberg A (1997) Concurrent positive and negative goal box events produce runway behaviors comparable to those of cocaine-reinforced rats. Pharmacol Biochem Behav 57:145-150

Grimm JW, Hope BT, Wise RA, Shaham Y (2001) Neuroadaptation: incubation of cocaine craving after withdrawal. Nature 412:141-142

Guzman D, Ettenberg A (2004) Heroin attenuates the negative consequences of cocaine in a runway model of selfadministration. Pharmacol Biochem Behav 79:317-324

Jaffe JH, Cascell NG, Kumor KM, Sherer MA (1989) Cocaineinduced cocaine craving. Psychopharmacology 97:59-64

Kelamangalath L, Wagner JJ (2009) Effects of abstinence or extinction on cocaine seeking as a function of withdrawal. Behav Pharmacol 20:195-203

Kippin TE, Fuchs RA, See RE (2006) Contributions of prolonged contingent and noncontingent cocaine exposure on enhanced reinstatement of cocaine seeking in rats. Psychopharmacology 187:60-70

Knackstedt LA, Ettenberg A (2005) Ethanol consumption reduces the adverse consequences of self-administered intravenous cocaine in rats. Psychopharmacol Berl 178:143-150

Knackstedt LA, Kalivas PW (2007) Extended access to cocaine selfadministration enhances drug-primed reinstatement but no behavioral sensitization. J Pharmacol Exp Ther 322:1103-1109

Knackstedt LA, Samimi MM, Ettenberg A (2002) Evidence for opponent-process actions of intravenous cocaine and cocaethylene. Pharmacol Biochem Behav 72:931-936

Kruzich PJ, Grimm JW, Rustay NR, Parks CD, See RE (1999) Predicting relapse to cocaine-seeking behavior: multiple regression approach. Behav Pharmacol 10:513-521

Leri F, Stewart J (2001) Drug-induced reinstatement to heroin and cocaine seeking: a rodent model of relapse in poly-drug use. Exp Clin Psychopharmacol 9:297-306

Lu L, Grimm JW, Shaham Y, Hope BT (2003) Molecular neuroadaptations in the accumbens and ventral tegmental area during the first 90 days of forced abstinence from cocaine selfadministration in rats. J Neurochem 85:1604-1613

Lu L, Grimm JW, Dempsey J, Shaham Y (2004) Cocaine seeking over extended withdrawal periods in rats: different time courses of responding induced by cocaine cues versus cocaine priming over the first 6 months. Psychopharmacology 176:101-108

Lundahl LH, Lukas SE (2007) Negative cocaine affect expectancies are associated with subjective response to cocaine challenge in recreational cocaine users. Addict Behav 32:1262-1271

Mantsch JR, Ho A, Schlussman SD, Kreek MJ (2001) Predictable individual differences in the initiation of cocaine self-administration by rats under extended-access conditions are dose dependent. Psychopharmacology 157:31-39
Mantsch JR, Yuferov V, Matheiu-Kia AM, Ho A, Kreek MJ (2004) Effects of extended access to high versus low cocaine doses on self-administration, cocaine-induced reinstatement and brain mRNA levels in rats. Psychopharmacology 175:26-36

McFarland K, Ettenberg A (1997) Reinstatement of drug-seeking behavior produced by heroin-predictive environmental stimuli. Psychopharmacology 131:86-92

Mendelson JH, Mello NK (1996) Management of cocaine abuse and dependence. N Engl J Med 334:965-972

Miller NE (1944) Experimental studies of conflict. In: Hunt IMcV (ed) Personality and the behavior disorders. Ronald, New York, pp 431-465

Nelson RA, Boyd SJ, Ziegelstein RC Herning R, Cadet JL, Henningfield JE et al (2005) Effect of rate of administration on subjective and physiological effects of intravenous cocaine in humans. Drug Alcohol Depend 82:19-24

O'Brien CP (1997) A range of research-based pharmacotherapies for addiction. Science 278:66-70

Raven MA, Necessary BD, Danluck DA, Ettenberg A (2000) Comparison of the reinforcing and anxiogenic effects of intravenous cocaine and cocaethylene. Exp Clin Pyschopharmacol 8:117-124

Rohsenow DJ, Niaura RS, Childress AR, Abrams DB, Monti P (1990) Cue-reactivity in addictive behaviors: theoretical and treatment implications. Int J Addict 25:957-993

Shaham Y, Funk D, Erb S, Brown TJ, Walker CD, Stewart J (1997) Corticotropin-releasing factor, but not corticosterone, is involved in stress-induced relapse to heroin-seeking in rats. J Neurosci 17:2605-2614

Shaham Y, Shalev U, Lu L, De Wit H, Stewart J (2003) The reinstatement model of drug relapse: history, methodology and major findings. Psychopharmacol Berl 168:3-20

Shalev U, Morales M, Hope B, Yap J, Shaham Y (2001) Timedependent changes in extinction behavior and stress-induced reinstatement of drug seeking following withdrawal from heroin in rats. Psychopharmacology 156:98-107

Tran-Nguyen LT, Fuchs RA, Coffey GP, Baker DA, O'Dell LE, Neisewander JL (1998) Time-dependent changes in cocaineseeking behavior and extracellular dopamine levels in the amygdala during cocaine withdrawal. Neuropsychopharmacology 19:48-59

Valles R, Rocha A, Nation JR (2006) The effects of acquisition training schedule on extinction and reinstatement of cocaine selfadministration in male rats. Exp Clin Psychopharmacol 14:245253

Van Dyke C, Byck R (1982) Cocaine. Sci Am 246:128-141

Ward SJ, Morgan D, Roberts DC (2005) Comparison of the reinforcing effects of cocaine and cocaine/heroin combinations under progressive ratio and choice schedules in rats. Neuropsychopharmacology 30:286-295

Weiss F, Martin-Fardon R, Cioccocioppo R, Kerr TM, Smith DL, Ben-Shahar O (2001) Enduring resistance to extinction of cocaine-seeking behavior induced by drug-related cues. Neuropsychopharmacology 25:361-372

Winger G, Skjoldager P, Woods JH (1992) Effects of buprenorphine and other opioid agonists and antagonists on alfentanil- and cocaine-reinforced responding rhesus monkeys. J Pharmacol Exp Ther 261:311-317

Withers NW, Pulbirenti L, Koob GF, Gillin JC (1995) Cocaine abuse and dependence. J Clin Psychopharmac 15:63-78

Zhou W, Xhang F, Liu H, Tang S, Lai M, Zhu H, Kalivas PW (2009) Effects of training and withdrawal periods on heroin seeking induced by conditioned cue in an animal model of relapse. Psychopharmacology 203:677-684 